\title{
Symmetry Breaking in Numeric Constraint Problems
}

\author{
Alexandre Goldsztejn ${ }^{1}$, Christophe Jermann ${ }^{1}$, \\ Vicente Ruiz de Angulo ${ }^{2}$, and Carme Torras ${ }^{2}$ \\ 1 Université de Nantes/CNRS LINA (UMR-6241) \\ 2 rue de la Houssinière, Nantes, F-44300 France. \\ name.surnameduniv-nantes.fr \\ 2 Institut de Robòtica i Informàtica Industrial (CSIC-UPC) \\ Llorens i Artigas 4-6, 08028-Barcelona, Spain. \\ surnamediri.upc.edu
}

\begin{abstract}
Symmetry-breaking constraints in the form of inequalities between variables have been proposed for a few kind of solution symmetries in numeric CSPs. We show that, for the variable symmetries among those, the proposed inequalities are but a specific case of a relaxation of the well-known LEX constraints extensively used for discrete CSPs. We discuss the merits of this relaxation and present experimental evidences of its practical interest.
\end{abstract}

Keywords: Symmetries, Numeric constraints, Variable symmetries

\section{Introduction}

Numeric constraint solvers are nowadays beginning to be competitive and even to outperform, in some cases, classical methods for solving systems of equations and inequalities over the reals. As a consequence, their application has raised interest in fields as diverse as neurophysiology and economics [18], biochemistry, crystallography, robotics [13] and, more generally, in those related to global optimization [9]. Symmetries naturally occur in many of these applications, and it is advisable to exploit them in order to reduce the search space and, thus, to increase the efficiency of the solvers.

Considerable work on symmetry breaking has been performed for discrete Constraint Satisfaction Problems (CSPs) in the last decades [7, 19]. Two main symmetrybreaking strategies have been pursued: 1) to devise specialized search algorithms that avoid symmetric portions of the search space [14, 8]; and 2) to add symmetry-breaking constraints (SBCs) that filter out redundant subspaces $[5,16]$. Contrarily to this, there exists very little work on symmetry breaking for numerical problems. For cyclic variables permutations, an approach divides the initial space into boxes and eliminates symmetric ones before the solving starts [17]. The addition of SBCs has also been proposed, but only for specific problems or specific symmetry classes, as inequalities between variables $[6,11,3]$.

In Section 2, we show that such inequalities are but a relaxation of the lexicographicordering based SBCs [4] widely used by the discrete CSP community. This relaxation allows us to generalize these previous works to any variable symmetry and can be derived automatically knowing the symmetries of a problem. In Section 3 we discuss its 
merits with respect to lexicographic-ordering based SBCs. In Section 4 we assess its practical interest. We provide tracks for future developments in Section 5.

\section{Symmetry-Breaking Constraints for NCSPs}

We are interested in solving the following general Numeric Constraint Satisfaction Problem (NCSP) $(X, D, C)$ : Find all points $X=\left(x_{1}, \ldots, x_{n}\right) \in D \subseteq \mathbb{R}^{n}$ satisfying the constraint $C(X)$, a relation on $\mathbb{R}^{n}$, typically a conjunction of non-linear equations and inequalities.

A function $s: \mathbb{R}^{n} \rightarrow \mathbb{R}^{n}$ is a symmetry of a NCSP if it maps bijectively solutions to solutions ${ }^{3}$, i.e., for all $X \in D$ such that $C(X)$ holds, $s(X) \in D$ and $C(s(X))$ also holds. In this case, we say $X$ and $s(X)$ are symmetric solutions, and by extension for any point $Y \in D, s(Y)$ is a symmetric point. We consider only symmetries that are permutations of variables. Let $\mathcal{S}_{n}$ be the set of all permutations of $\{1, \ldots, n\}$. The image of $i$ by a permutation $\sigma$ is $i^{\sigma}$, and $\sigma$ is described by $\left[1^{\sigma}, 2^{\sigma}, \ldots, n^{\sigma}\right]$. A symmetry $s$ is a variable symmetry iff there is a $\sigma \in \mathcal{S}_{n}$ such that for any $X \in D, s(X)=$ $\left(x_{1^{\sigma}}, \ldots, x_{n^{\sigma}}\right)$. We identify such symmetries with their associated permutations and denote both by $\sigma$ in the following. Consequently, the set of variable symmetries of a NCSP is isomorphic to a permutation subgroup of $\mathcal{S}_{n}$, which are both identified and denoted by $\Sigma$ in the following.

Example 1. The 3-cyclic roots problem is: find all $\left(x_{1}, x_{2}, x_{3}\right) \in \mathbb{R}^{3}$ satisfying $\left(x_{1}+\right.$ $\left.x_{2}+x_{3}=0\right) \wedge\left(x_{1} x_{2}+x_{2} x_{3}+x_{3} x_{1}=0\right) \wedge\left(x_{1} x_{2} x_{3}=1\right)$. This problem has six variable symmetries including identity, $\Sigma=\{[1,2,3]$, [1,3, 2], $[2,1,3],[2,3,1],[3,1,2]$, $[3,2,1]\}$. Hence, all its variables are interchangeable.

We say that the symmetries of a CSP are completely broken when a single representative in each set of symmetric solutions is retained. To this end, it is possible to add symmetry-breaking constraints (SBCs) which will exclude all but a single representative of the symmetric solutions [7, 19]. Crawford et al. [4] proposed lexicographic ordering constraints (LEX) that completely break any variable symmetry. Recall that given $X$ and $Y$ both in $\mathbb{R}^{n}$ the lexicographic order is defined inductively as follows:

$$
\begin{aligned}
& \text { for } n=1, \quad X \preceq_{\text {lex }} Y \equiv\left(x_{1} \leq y_{1}\right) \\
& \text { for } n>1, \quad X \preceq_{\text {lex }} Y \equiv\left(x_{1}<y_{1}\right) \vee\left(\left(x_{1}=y_{1}\right) \wedge\left(X_{2: n} \preceq_{l e x} Y_{2: n}\right)\right)
\end{aligned}
$$

where $X_{2: n}=\left(x_{2}, \ldots, x_{n}\right)$, and the same for $Y$. For a given symmetry $\sigma$, Crawford $e t$ $a l$. define the corresponding $\operatorname{SBC}_{\operatorname{LEX}}(X) \equiv X \preceq_{\text {lex }} \sigma(X)$. Intuitively, this constraint imposes a total order on the symmetric solutions, hence allowing to retain a single one w.r.t. a given symmetry $\sigma$. One such constraint is thus imposed for each of the symmetries of a problem in order to break them all. The strength of these constraints is that they reduce the search space by a factor equal to $\# \Sigma$, the order of the symmetry group $\Sigma$ of the problem. One critical issue however is that the number of SBCs can be exponential with respect to the number of variables.

\footnotetext{
${ }^{3}$ Nothing is required for non-solution points, i.e., we consider solution symmetries [1].
} 
Example 2. Excluding the identity permutation, a symmetry of any problem which is irrelevant to break, the LEX constraints for the symmetries of the 3 -cyclic-roots problem are: $\left(x_{1}, x_{2}, x_{3}\right) \preceq_{\text {lex }}\left(x_{1}, x_{3}, x_{2}\right),\left(x_{1}, x_{2}, x_{3}\right) \preceq_{\text {lex }}\left(x_{2}, x_{1}, x_{3}\right),\left(x_{1}, x_{2}, x_{3}\right) \preceq_{\text {lex }}$ $\left(x_{2}, x_{3}, x_{1}\right),\left(x_{1}, x_{2}, x_{3}\right) \preceq_{\text {lex }}\left(x_{3}, x_{1}, x_{2}\right)$, and $\left(x_{1}, x_{2}, x_{3}\right) \preceq_{\text {lex }}\left(x_{3}, x_{2}, x_{1}\right)$.

Since they offer a good trade-off between the solving time reduction they allow, and the difficulty to handle them, partial SBCs (PSBCs), that retain at least one representative of the symmetric solutions, have often been considered. Especially for NCSPs, several classes of variable symmetries have been broken using PSBCs having the form of inequalities between variables. For instance, Gasca et al. [6] proposed PSBCs $x_{i} \leq x_{i+1}(i \in\{1, \ldots, n-1\})$ for full permutations $\left(\Sigma=\mathcal{S}_{n}\right)$, and PSBCs $x_{1} \leq x_{i}$ $(i \in\{2, \ldots, n\})$ for cyclic permutations $\left(\Sigma=\mathcal{C}_{n}\right)^{4}$. Similar PSBCs have been proposed for numeric optimization problems with more peculiar symmetry groups, e.g., $\Sigma=\mathcal{C}_{2} \times \mathcal{S}_{n}$ in [3] and $\Sigma=\prod_{i} \mathcal{S}_{p_{i}}$ in [11].

Example 3. Considering again the 3-cyclic-roots problem, Gasca et al.'s PSBCs are: $x_{1} \leq x_{2}$ and $x_{2} \leq x_{3}$. Indeed, these inequalities filter out all but a single of the six symmetries of any solution to this problem.

The corner stone of our approach is to note that all the PSBCs mentioned above can be obtained by relaxing Crawford's SBCs as follows: For $\sigma \in \mathcal{S}_{n}$ different from the identity permutation, and $X=\left(x_{1}, \ldots, x_{n}\right)$, we define the constraint $\operatorname{RLEX}_{\sigma}(X) \equiv$ $x_{k_{\sigma}} \leq x_{k_{\sigma} \sigma}$, where $k_{\sigma}$ is the smallest integer in $\{1, \ldots, n\}$ such that $k_{\sigma} \neq k_{\sigma}{ }^{\sigma}$. The following proposition establishes that this constraint is a relaxation of a LEX constraint, i.e., a PSBC: it cannot remove any solution preserved by LEX constraint.

Proposition 1. $\operatorname{LEX}_{\sigma}(X) \Longrightarrow \operatorname{RLEX}_{\sigma}(X)$

Proof. Since $i<k_{\sigma}$ implies $i=i^{\sigma}$, we have $x_{i}=x_{i^{\sigma}}$ for all $i<k_{\sigma}$. Therefore $\operatorname{LEX}_{\sigma}(X)$, which is $X \preceq_{l e x} \sigma(X)$, is actually equivalent to $X_{k_{\sigma}: n} \preceq_{l e x} \sigma(X)_{k_{\sigma}: n}$, i.e.,

$$
\left(x_{k_{\sigma}}<x_{k_{\sigma} \sigma}\right) \vee\left(\left(x_{k_{\sigma}}=x_{k_{\sigma} \sigma}\right) \wedge\left(X_{k_{\sigma}+1: n} \preceq_{l e x} \sigma(X)_{k_{\sigma}+1: n}\right)\right),
$$

which logically implies $\left(x_{k_{\sigma}}<x_{k_{\sigma} \sigma}\right) \vee\left(x_{k_{\sigma}}=x_{k_{\sigma} \sigma}\right)$, that is $\operatorname{RLEX}_{\sigma}(X)$.

The ad-hoc inequalities proposed so far to partially break specific classes of variable symmetries in NCSPs are just special cases of the RLEX constraints. For instance, when $\Sigma=\mathcal{S}_{n}$, Gasca et al.'s PSBCs are $x_{i} \leq x_{i+1}(i \in\{1, \ldots, n-1\})$ [6]. In this case, $k_{\sigma}$ takes all possible values in $\{1, \ldots, n-1\}$ and $k_{\sigma}{ }^{\sigma}$ all possible values in $\left\{k_{\sigma}+1, \ldots, n\right\}$. Hence the corresponding RLEX constraints are $x_{i} \leq x_{j}(i<j)$. Since all the inequalities $x_{i} \leq x_{j}$ with $i+1<j$ among them are redundant, they can be eliminated, yielding the inequalities proposed by Gasca et al. A similar verification is easily carried out for the other specific variables symmetries tackled in $[6,3,11]$. Hence, RLEX constraints generalize these PSBCs to any variable symmetries.

Example 4. Continuing Example 2, the corresponding RLEX constraints are respectively: $x_{2} \leq x_{3}, x_{1} \leq x_{2}, x_{1} \leq x_{2}, x_{1} \leq x_{3}$ and $x_{1} \leq x_{3}$. This set of inequalities can be simplified to $x_{1} \leq x_{2}$ and $x_{2} \leq x_{3}$, i.e., that presented in Example 3 .

\footnotetext{
${ }^{4} \mathcal{C}_{n}=\{[k, \ldots, n, 1, \ldots, k-1]: k \in\{1, \ldots, n\}\}$
} 


\section{RLEX VS LEX}

Advantages. First, we draw the reader's attention to the simplicity of the relaxed constraints w.r.t. the original ones: RLEX constraints are just binary inequalities while LEX constraints involve all the variables of the symmetries in a large combination of logical operations. Hence, we expect it is much more efficient to prune RLEX constraints (no specific algorithm is required) and to propagate the obtained reductions (successful reductions trigger only constraints depending on two variables), than LEX constraints.

Second, and more prominently, the number of RLEX constraint is always smaller than the number of LEX constraints, and it is bounded upward by $\frac{n(n-1)}{2}$ (number of different pairs $\left(x_{i}, x_{j}\right)$ with $i<j$ ), or only $n-1$ if one considers a non-redundant subset of inequalities as we explained previously. In contrast, there can be exponentially many LEX constraints, one for each permutation in $\mathcal{S}_{n}$. As remarked by Crawford et al., this makes the use of LEX constraints impractical in general and has yielded research towards simplifying and relaxing them [4]. Oppositely, adding $O(n)$ RLEX constraints to a CSP model should never be a problem for its practical treatment by a solver.

Similar constraints $x_{k_{\sigma}}<x_{k_{\sigma}}$ were proposed by Puget in [15] as SBCs for (discrete) problems where the variables are subject to an all different constraint. It is thus possible to obtain the RLEX constraints without having to compute all LEX constraints by applying the group theory results already used by Puget: From a generating set of the symmetries $\Sigma$ of a problem, it is possible to derive a stabilizer chain, i.e., a sequence of permutation subgroups such that each is contained in the preceding and the permutations in the $i^{\text {th }}$ subgroup map all integers in $\{1, \ldots, i\}$ to themselves. The orbit of the integer $i+1$ in the $i^{t h}$ subgroup, i.e., all the integers it can be mapped to by any permutation in this subgroup, thus define exactly the pairs for which we must impose an inequality. These pairs can be obtained with the Shreier-Sims algorithm which runs in $O\left(n^{2} \log ^{3}(\# \Sigma)+t n \log (\# \Sigma)\right)$, where $t$ is the cardinality of the input generating set $^{5}$. Since $\# \Sigma$ is at most $n$ ! (when $\Sigma=\mathcal{S}_{n}$ ), this algorithm runs in polynomial time in $n$ and $t$.

Hence, RLEX constraints constitute a generalization of the inequalities proposed so far for NCSPs that remains of tractable size and can be computed in polynomial time for any variable symmetries.

Drawbacks. The RLEX constraints break only partially the symmetries that LEX constraints break completely. Let us describe more precisely symmetric solutions which are discarded by LEX but not by RLEX.

Given a symmetry $\sigma$ and a solution $X=\left(x_{1}, \ldots, x_{n}\right)$, if $\sigma(X)$ is discarded by the corresponding LEX constraint, it means that there exists $i$ such that $x_{i}<x_{i^{\sigma}}$ and $\forall j \in\{1, \ldots, i-1\}, x_{j}=x_{j^{\sigma}}$. If $\sigma(X)$ is not discarded by the corresponding RLEX constraint $x_{k_{\sigma}} \leq x_{k_{\sigma} \sigma}$, it means that $k_{\sigma}<i$. Thus, $x_{k_{\sigma}}=x_{k_{\sigma}{ }^{\sigma}}$ while $k_{\sigma} \neq k_{\sigma}{ }^{\sigma}$ by definition, i.e., $X$ must lie on a given hyperplane $H_{u v}=\left\{X \mid x_{u}=x_{v}\right\}$.

Hence, all the symmetric solutions that are discarded by LEX constraints (w.r.t. all the symmetries of the problem) but not by RLEX constraints belong to such hyperplanes. Because the volume of these hyperplanes is null in $\mathbb{R}^{n}$, the set of points filtered out by

\footnotetext{
${ }^{5}$ A minimal generating set is $O(n)$ for any subgroup of variable symmetries.
} 
LEX constraints and preserved by RLEX constraints represents a null volume of the search space. We conclude that RLEX constraints reduce the search space volume by a factor $\# \Sigma$ identical to that achieved with LEX constraints.

Moreover, numerical constraint solvers cannot eliminate these singular symmetric solutions even with LEX constraints since they do not distinguish strict and non-strict inequalities. Indeed, they perform computations using intervals and thus cannot approximate open sets differently from closed ones.

In conclusion, since the aim of PSBCs is essentially to enhance the solvers performances by allowing quick and easy reduction of the search space, it appears RLEX constraints are a very good trade-off between simplicity and efficiency: they are easy to derive, simple to handle, and still filter out most of the symmetric search space.

\section{Experimental results}

We provide experimental evidences of the important performance gains RLEX constraints can bring when solving symmetric NCSPs. Indeed, the solving time of a given NCSP is in general proportional to its search space. We expect RLEX constraints allow to quickly eliminate large portions of the search space, isolating an asymmetric subsearch space whose volume is divided by $\# \Sigma$ w.r.t. the initial search space. As a result, we expect to observe computation time gains proportional to $\# \Sigma$.

All experiments are conducted on a dual-core equipped machine $(2.5 \mathrm{GHz}, 4 \mathrm{~Gb}$ RAM) using the Realpaver [10] constraint solver with default settings.

Preliminar analysis: We first consider homemade scalable problems whose solutions either lie outside any hyperplane $H_{u v}$ (problems $P_{1}, P_{2}$ ), all lie on such hyperplanes (problems $P_{3}, P_{4}$ ), or lie at the intersection of all these hyperplanes (problems $P_{5}$, $\left.P_{6}\right)$. In all cases, we consider problems with only cyclic permutations $\left(P_{1}, P_{3}, P_{5}\right)$ and others with full permutations $\left(P_{2}, P_{4}, P_{6}\right)$, i.e., problems for which the volume of the asymmetric search space is $\frac{1}{n}$ and $\frac{1}{n !}$ of that of the initial search-space respectively :

$$
\begin{aligned}
& \mathbf{P}_{\mathbf{1}}: X \in[-n, n]^{n}, \prod_{\sigma \in \mathcal{C}_{n}}\left\|\sigma(X)-X^{*}\right\|=0 \\
& \mathbf{P}_{\mathbf{2}}: X \in[-n, n]^{n}, \prod_{\sigma \in \mathcal{S}_{n}}\left\|\sigma(X)-X^{*}\right\|=0 \\
& \mathbf{P}_{\mathbf{3}}: X \in[-2,2]^{n}, \prod_{j=1}^{n}\left(\sum_{i=1}^{n}\left(x_{((i+j) \bmod n)}+(-1)^{i}\right)^{2}\right)=0 \\
& \mathbf{P}_{\mathbf{4}}: X=\in[0,1]^{n}, \forall i \in 1 . . n \sum_{j \neq i} x_{j}^{2}+x_{i} \cos \left(\sum_{j=1}^{n} x_{j}\right)=0 \\
& \mathbf{P}_{\mathbf{5}}: X \in[-2,2]^{n}, \sum_{i=1}^{n}\left(x_{i}^{2}-1\right)^{2}=0 \\
& \mathbf{P}_{\mathbf{6}}: X \in[0,1]^{n}, \forall i \in 1 . . n \sum_{j=1}^{n-1}\left(\prod_{l=1}^{n-1} x_{(i+j+l) \operatorname{modn} n}\right)=1
\end{aligned}
$$

where $X^{*}$ is the point $(1, \ldots, n) \in \mathbb{R}^{n}$. The solutions of $P_{1}$ are all cyclic permutations of $X^{*}$ while that of $P_{2}$ are all permutations of $X^{*}$. The solutions of $P_{3}$ are the cyclic permutations ${ }^{6}$ of $(-1,1, \ldots,-1,1) \in \mathbb{R}^{n}$; that of $P_{4}$ are all points of the form $\{-1,1\}^{n} \cdot P_{5}$ and $P_{6}$ both have a single (very symmetric) solution: $0^{n}$.

\footnotetext{
${ }^{6}$ Note there are only 2 different solutions when $n$ is even, $n$ solutions when it is odd.
} 


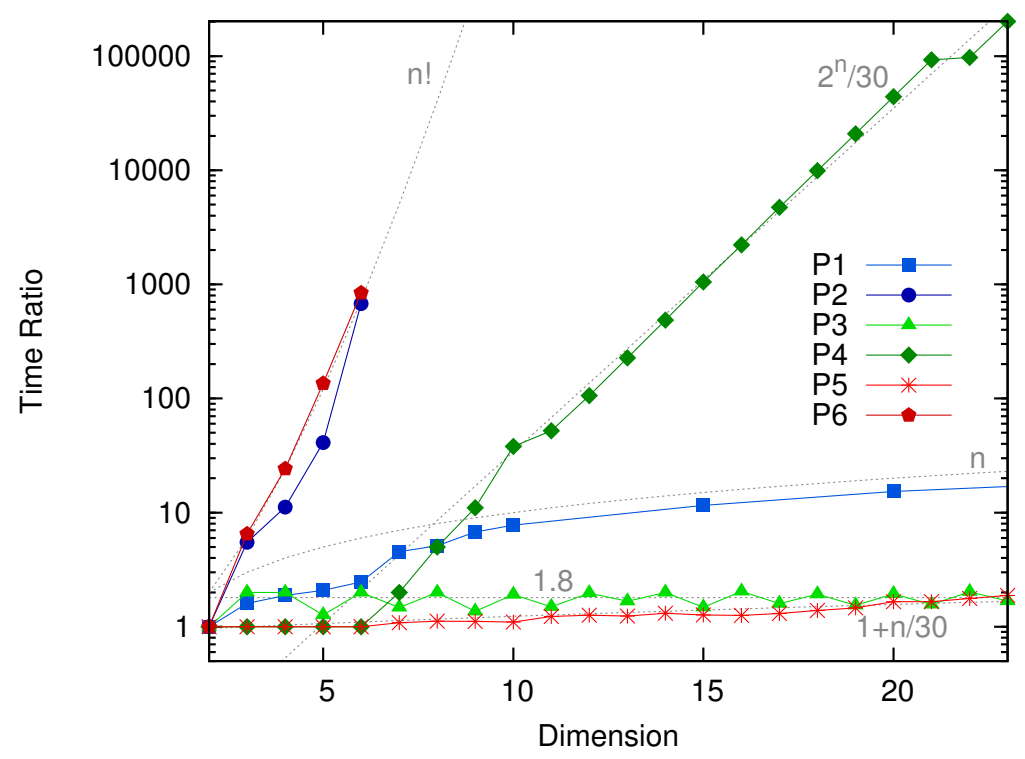

Fig. 1. Time ratios for homemade problems

Figure 1 presents the variation of the ratio between the computation time without RLEX and the computation time with it (called gain in the following) when the dimension $n$ varies. In addition to the measured gains, the figure displays (in dotted gray) the functions of $n$ that best approximate them.

The gains for $P_{1}$ and $P_{2}$ follow very closely the reduction factor of their search space volume, hence confirming our expectations. Note that although the gains are not as impressive for $P_{1}$ as for $P_{2}$, they are already significant: E.g., for $n=50$ the computation time is $1124 \mathrm{~s}$ ( $>18 \mathrm{~min}$ ) without RLEX and 29s with RLEX. For $P_{2}$ they are really outstanding: E.g., for $n=6$, the computations time is $12863 \mathrm{~s}$ ( $>3.5 \mathrm{~h})$ without RLEX but only 19 s with RLEX.

For the other problems, the results are more varied: $P_{3}$ presents only an (almost) constant gain; $P_{4}$ shows a gain closer to the reduction factor of the size of its solution set than to its search space volume reduction factor; $P_{5}$ offers a (quite flat) linear gain, i.e., proportional to its search space volume reduction factor; the gain for $P_{6}$ follows closely its search space volume reduction factor ${ }^{7}$. The factors that could explain this diversity of behaviors are numerous (e.g., relative pruning power of the original constraints w.r.t. the added PSBCs, proportion of symmetric solutions with and without RLEX, ...). Further experiments will be necessary to distinguish the exact effects of all these factors.

The conclusion we draw from these results is that one cannot always expect as much gain as the search space volume reduction factor, especially when the problem

\footnotetext{
${ }^{7}$ Computations for $P_{6}$ could not be performed further because the timings were becoming too large, e.g., $41751 \mathrm{~s}$ ( $>11.5 \mathrm{~h}$ ) for $n=6$ without RLEX, as compared to 49.5 s with it.
} 


\begin{tabular}{|l|r|c|c|c|c|r|}
\hline Problem & $n$ & Sol & $\# \Sigma$ & $\begin{array}{c}\text { Time } \\
\text { w/RLEX }\end{array}$ & $\begin{array}{c}\text { Time } \\
\text { w RLEX }\end{array}$ & gain \\
\hline Brown & 5 & $\mathrm{~S}$ & $n !$ & 0.95 & 0.24 & 3.9 \\
& 8 & & & 1218 & 5.32 & 229.0 \\
\hline Cyclic & 4 & $\mathrm{GS}^{*}$ & $2 n$ & 260 & 32.1 & 8.1 \\
roots & 5 & $\mathrm{~S}$ & & 46.6 & 4.7 & 9.7 \\
& 6 & $\mathrm{~S}$ & & 2017 & 183 & 10.9 \\
\hline Cyclohexane & 3 & $\mathrm{~S}$ & $n !$ & 0.24 & 0.16 & 1.5 \\
\hline Extended & 20 & $\mathrm{~S}$ & $\frac{n}{2} !$ & 0.41 & 0.26 & 1.6 \\
Freudenstein & 140 & & & 422 & 315 & 1.3 \\
\hline Extended & 16 & $\mathrm{~S}$ & $n \frac{n}{2} !$ & 1.42 & 0.03 & 47.3 \\
Powell & 30 & & & 844 & 0.1 & 8442.0 \\
\hline Feigenbaum & 11 & $\mathrm{GS}$ & $n$ & 7.30 & 0.81 & 9.0 \\
& 23 & & & 10924 & 1027 & 10.6 \\
\hline
\end{tabular}

Table 1. Results for various problems from the literature

has singular solutions; still, the gains can be outstanding, and adding RLEX constraints did not induce any uncompensated overhead in any of the settings we have considered.

Standard benchmark: We also consider a benchmark composed of standard problems picked from [2]. Their characteristics and the results obtained are reported in Table 1. For scalable problems we report timings for the smallest and largest dimension $n$ we tested, allowing one to imagine the gain variation with the dimension. Column "Sol" indicates the type of solutions of the problem: $G=$ Generic (i.e., out of any hyperplane $\left.H_{u v}\right)$ and $S=$ Singular. Note that most of these standard problems are of type $S$. Problem 4-cyclic-roots is marked $G S^{*}$ because this problem has a continuous solution set which intersects some $H_{u v}$ hyperplanes. For this problem, timings correspond to paving its solution manifold with $10^{-2}$-wide boxes.

For problems Brown, Cyclic-roots and Extended-Powell, the gain closely follows the search space volume reduction factor (column $\# \Sigma$ ). Still, for problems ExtendedFreudenstein and Feigenbaum the gains remain almost constant as the dimension grows. These experiments support the preliminary analysis we have performed: We can achieve important gains for highly symmetric problems and the introduction of RLEX constraints at least does not appear counterproductive.

\section{Conclusion and Future Prospects}

We have presented a generalization of the PSBCs proposed so far for variable symmetries in NCSPs. It corresponds to a relaxation of the famous LEX constraints used for breaking symmetries essentially for discrete CSPs so far. We have discussed the merits of this relaxation w.r.t. LEX constraints and illustrated its practical interest for NCSPs.

All the arguments we have used are also valid for continuous optimization and constrained optimization problems. Considering that many of them are not specific to numeric problems or solvers, it would also be interesting to consider this relaxation 
in discrete domains. Hence, we should also consider Mixed-Integer Linear/Nonlinear Programming and Integer Linear Programming where some of the PSBCs we have generalized have been proposed $[3,12]$.

\section{References}

1. Cohen, D., Jeavons, P., Jefferson, C., Petrie, K., Smith, B.: Symmetry definitions for constraint satisfaction problems. Constraints 11(2-3), 115-137 (2006)

2. COPRIN: The inria project COPRIN examples webpage. http: //www-sop.inria.fr/coprin/logiciels/ALIAS/Benches/ (2011)

3. Costa, A., Liberti, L., Hansen, P.: Formulation symmetries in circle packing. Electronic Notes in Discrete Mathematics 36, 1303 - 1310 (2010)

4. Crawford, J., Ginsberg, M., Luks, E., Roy, A.: Symmetry-breaking predicates for search problems. In: KR. pp. 148-159 (1996)

5. Flener, P., Frisch, A., Hnich, B., Kiziltan, Z., Miguel, I.: Breaking row and column symmetries in matrix models. In: $\mathrm{CP}$ '02: Proceedings of the 8th International Conference on Principles and Practice of Constraint Programming. pp. 462-476. Springer (2002)

6. Gasca, R., Valle, C.D., Cejudo, V., Barba, I.: Improving the computational efficiency in symmetrical numeric constraint satisfaction problems. In: 11th Conference of the Spanish Association for Artificial Intelligence (CAEPIA). pp. 269-279 (2005)

7. Gent, I., Petrie, K., Puget, J.F.: Handbook of Constraint Programming, chap. Symmetry in constraint programming, pp. 329-376. Elsevier (2006)

8. Gent, I.P.: Groups and constraints: Symmetry breaking during search. In: In Proceedings of CP-02, LNCS 2470. pp. 415-430. Springer (2002)

9. Goldsztejn, A., Lebbah, Y., Michel, C., Rueher, M.: Capabilities of constraint programming in safe global optimization. In: International Symposium on Nonlinear Theory and its Applications. pp. 601-604 (2008)

10. Granvilliers, L., Benhamou, F.: Algorithm 852: Realpaver: an interval solver using constraint satisfaction techniques. ACM T. on Mathematical Software 32, 138-156 (2006)

11. Ji, X., Ma, F., Zhang, J.: Solving global unconstrained optimization problems by symmetrybreaking. In: $8^{\text {th }}$ IEEE/ACIS International Conference on Computer and Information Science. pp. 107-111 (2009)

12. Margot, F.: Symmetry in integer linear programming. In: 50 Years of Integer Programming 1958-2008, pp. 647-686. Springer (2010)

13. Merlet, J.P.: Interval analysis for certified numerical solution of problems in robotics. Applied Mathematics and Computer Science 19(3), 399-412 (2009)

14. Meseguer, P., Torras, C.: Exploiting symmetries within constraint satisfaction search. Artif. Intell. 129(1-2), 133-163 (2001)

15. Puget, J.F.: Breaking symmetries in all different problems. In: Proc. 19th international joint conference on Artificial intelligence (IJCAI). pp. 272-277 (2005)

16. Puget, J.F.: Symmetry breaking revisited. Constraints 10(1), 23-46 (2005)

17. Ruiz de Angulo, V., Torras, C.: Exploiting single-cycle symmetries in continuous constraint problems. Journal of Artificial Intelligence Research 34, 499-520 (2009)

18. Vu, X.H., Schichl, H., Sam-Haroud, D.: Interval propagation and search on directed acyclic graphs for numerical constraint solving. J. Global Optimization 45(4), 499-531 (2009)

19. Walsh, T.: Parameterized complexity results in symmetry breaking. In: Parameterized and Exact Computation, proc. $5^{\text {th }}$ International Symposium IPEC. Springer LNCS, vol. 6478, pp. 4-14 (2010) 